\title{
Robust AOA based acoustic source localization method with unreliable measurements
}

\author{
Qingli Yan ${ }^{\mathrm{a}, \mathrm{b}, *}$, Jianfeng Chen ${ }^{\mathrm{a}}$, Geoffrey Ottoy ${ }^{\mathrm{b}}$, Lieven De Strycker ${ }^{\mathrm{b}}$ \\ a School of Marine Science and Technology, Northwestern Polytechnical University, China \\ ${ }^{\mathrm{b}} \mathrm{KU}$ Leuven, ESAT-DRAMCO, Ghent Technology Campus, Ghent 9000, Belgium
}

\section{A R T I C L E I N F O}

\section{Article history:}

Received 28 November 2017

Revised 11 May 2018

Accepted 12 May 2018

Available online 15 May 2018

\section{Keywords:}

Acoustic source localization

M-estimator

Angle of arrival

Unreliable measurements

\begin{abstract}
A B S T R A C T
The performance degradation problem of the angle of arrival (AOA)-based acoustic localization methods in the presence of unreliable bearing measurements (outliers) is addressed in this paper. Two typical Mestimators based on Tukey and Huber functions are applied to tackle the problem. Both functions are solved by the iterative reweighted nonlinear least squares (IRNLS) method. Considering the Huber function is convex in nature, it is specifically utilized to mitigate the influence of large residuals on pseudolinear estimator (PLE) by convex optimization. To make the IRNLS method more feasible to use, an approximate relationship between the outlier probability and the bound parameter is provided. The robustness and effectiveness of the proposed methods are clearly demonstrated through a series of simulation results in the presence of various unreliable measurements.
\end{abstract}

(c) 2018 Elsevier B.V. All rights reserved.

\section{Introduction}

The angle of arrival (AOA), also named bearing, based acoustic source localization technique has been successfully applied in many promising applications, such as security, telecommunication and environmental monitoring [1-3]. As a hot research topic, many effective approaches have been proposed, such as maximum likelihood estimator (MLE) [4], pseudolinear estimator (PLE) [5], reduced-bias PLE [6], approximate maximum likelihood (AML) [7], extended Kalman filtering [8,9] and total least squares (TLS) [10]. Among these methods, MLE and PLE are most commonly used methods. MLE is an asymptotically unbiased and efficient estimator, but it does not have a closed-form solution and has to be implemented in an iterative search way [4]. PLE which does not require a Gaussian noise assumption is simple to implement with lower complexity. Though PLE suffers from bias, it can be overcome by the bias compensation methods [6].

With the progresses in sensor technology, people start to uses a large amount of low cost sensor nodes for sound source localization in unknown fields [11,12]. Theoretically speaking, if the background noise in the field follows the Gaussian distribution, both PLE and MLE perform well, even though Gaussian noise is not always strictly required by PLE. However, the distributed sensors often encounter unexpected interferences [13], such as external attacks, natural causes (wind or storm for example), or sensor fail-

\footnotetext{
* Corresponding author.

E-mail address: gongchyy@163.com (Q. Yan).
}

ures, etc. On the other hand, the low cost sensor nodes are usually made with stringent resource in transmission power, AD sampling resolution, or computational capacity, etc. These interferences and limitations may result in many unreliable measurements or called outliers. As a result, the above-mentioned traditional AOAbased methods fail to provide reasonable estimates when outliers are present.

One way to solve outlier problem is to identify them, then remove them. A least squares estimator that leverages the hybrid estimate method of AOA and TOA has been used to detect the NLOS signal [14]. By assuming that the reliable sensors are expected to behave similarly, the Bayesian method is proposed to identify unreliable sensors from a given set of sensors [15]. A distributed localization approach based on expectation maximization (EM) method is developed when sensor failure happens [16]. The EM based method is also introduced to attain the accurate localization results for AOA based localization method by identifying the unreliable measurements from NLOS propagation environment [17]. The unreliable bearing measurements method is presented by detecting the outliers from a set of estimated positions obtained by different sensor combinations [18].

Another typical way is incorporating all the measurements into a robust estimator against outliers. A well-known robust estimator is the M-estimator, which has been widely used to process outlier problems for robust registration of point sets [19], linear regression [20] and robust estimation of diffusion magnetic resonance parameters [21]. For acoustic source localization problems, a bi-square M-estimation has been proposed to estimate the acoustic source 


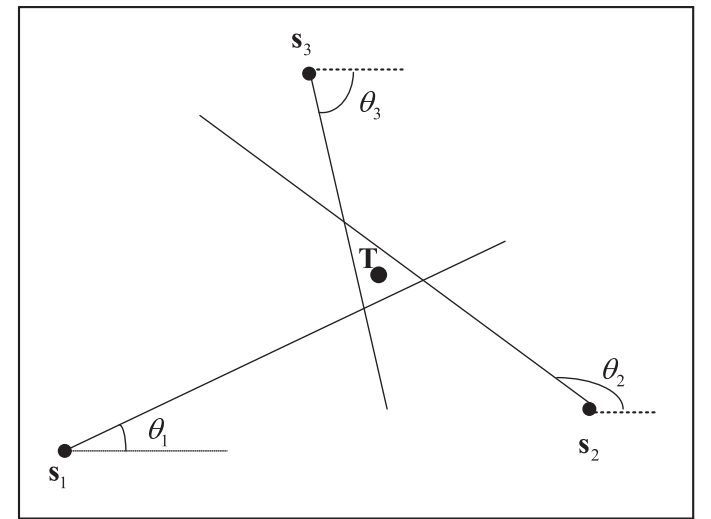

Fig. 1. I Illustration of an AOA localization system using three sensors.

location with outlier-corrupted sensor observations in an energybased localization method [22]. The M-estimator has been introduced into the extended Kalman filter based on the time difference of arrival (TDOA) method to solve the NLOS mitigation problem [23]. Chen describes a robust least-mean-M-estimate algorithm to estimate the time delay in case of multichannel propagation [24]. Note that specific methods to estimate the accurate AOAs for nonGaussian channel before localization are outside the scope of this paper; interested readers are referred to [25-29].

The motivation of this paper is to propose a robust localization method to reduce the influence of unreliable AOA measurements caused by uncertain environments. The main contributions can be summarized as follows:

- The performances of the traditional localization methods using the Non-Linear Estimator (NLE) and the Pseudo Linear Estimator (PLE) are analyzed when these estimators are subject to the AOA outliers.

- Two estimators based on Tukey and Huber function, respectively, are proposed to decrease the influence of the AOA outliers for NLE, and Huber function is addressed for HPLE.

- To make the new estimators easy to use, a threshold determination method based on the outlier probability is designed and verified through a series of simulations.

This paper is organized as follows. In Section 2, the traditional AOA based localization methods are reviewed, with an emphasis on the influence of unreliable bearings on the localization performance. Section 3 provides the uncertain model for AOA measurements. Both the Tukey and Huber estimators are proposed to solve the outlier problem for NLE, the Huber function is also applied to solve the outlier problem for PLE. Section 4 presents the proper bound parameter determination method for the proposed methods; the localization performances of our proposed methods and several traditional methods are compared using numerical experiments. The conclusions are discussed in Section 5.

\section{AOA based localization methods and problem formulation}

\subsection{Angle of arrival model with gaussian noise}

As shown in Fig. 1, in this paper, we consider an acoustic localization system with a single stationary acoustic source located at $\mathbf{T}=[x, y]^{\mathrm{T}}$ and multiple sensors, located at $\mathbf{s}_{k}=\left[x_{k}, y_{k}\right]^{\mathrm{T}}, k=1,2, \ldots$, $N$. Ideally, the true bearing $\theta_{k}$ can be expressed as:

$\theta_{k}=\arctan \left(\frac{y-y_{k}}{x-x_{k}}\right), k=1, \ldots, N$.

The observations are usually subject to measurement noise:

$\hat{\theta}_{k}=\theta_{k}+\varepsilon_{k}, k=1, \ldots, N$, where $\varepsilon_{k}$ are assumed to be independent zero-mean Gaussian noise with variance $\sigma_{k}^{2}$. The set of measurements from $N$ sensors can be written in vector form as

$\hat{\boldsymbol{\theta}}=\boldsymbol{\theta}+\boldsymbol{\varepsilon}$,

where $\hat{\boldsymbol{\theta}}=\left[\begin{array}{llll}\hat{\theta}_{1} & \hat{\theta}_{2} & \ldots & \hat{\theta}_{N}\end{array}\right]^{\mathrm{T}}, \boldsymbol{\theta}=\left[\begin{array}{llll}\theta_{1} & \theta_{2} & \ldots & \theta_{N}\end{array}\right]^{\mathrm{T}}$, and $\boldsymbol{\varepsilon}=$ $\left[\begin{array}{llll}\varepsilon_{1} & \varepsilon_{2} & \ldots & \varepsilon_{N}\end{array}\right]^{\mathrm{T}}$.

\subsection{Maximum likelihood estimator and its outlier effect}

Under the Gaussian noise assumption, the likelihood function of the bearing observations can be given by

$f(\hat{\boldsymbol{\theta}} \mid \mathbf{T})=\frac{1}{(2 \pi)^{N / 2}|\mathbf{Q}|^{1 / 2}} \exp \left\{-\frac{1}{2}(\hat{\boldsymbol{\theta}}-\boldsymbol{\theta}(\mathbf{T}))^{\mathrm{T}} \mathbf{Q}^{-1}(\hat{\boldsymbol{\theta}}-\boldsymbol{\theta}(\mathbf{T}))\right\}$,

where $\mathbf{Q}$ is the $N \times N$ covariance matrix of bearing observation errors

$\mathbf{Q}=\operatorname{diag}\left(\sigma_{1}^{2}, \sigma_{2}^{2}, \ldots, \sigma_{N}^{2}\right)$.

The target position, $\hat{\mathbf{T}}_{M L}$, can be obtained by maximizing the likelihood function (4). Typically, the log- function of (4) is used to simplify the maximization problem:

$\ln f(\hat{\boldsymbol{\theta}} \mid \mathbf{T})=-\frac{1}{2} \ln \left((2 \pi)^{N}|\mathbf{T}|\right)-\frac{1}{2}(\hat{\boldsymbol{\theta}}-\boldsymbol{\theta}(\mathbf{T}))^{\mathrm{T}} \mathbf{Q}^{-1}(\hat{\boldsymbol{\theta}}-\boldsymbol{\theta}(\mathbf{T}))$.

The maximization of (6) can be obtained by minimizing the second term of (6) [4]

$\hat{\mathbf{T}}_{M L}=\arg \min L_{M L}(\mathbf{T})$,

where

$L_{M L}(\mathbf{T})=(\hat{\boldsymbol{\theta}}-\boldsymbol{\theta}(\mathbf{T}))^{\mathrm{T}} \mathbf{Q}^{-1}(\hat{\boldsymbol{\theta}}-\boldsymbol{\theta}(\mathbf{T}))$.

A simple gradient-descent approach can be used to solve (8) recursively:

$\mathbf{T}(k+1)=\mathbf{T}(k)-\left.\mu \frac{\partial L_{M L}(\mathbf{T})}{\partial \mathbf{T}}\right|_{\mathbf{x}=\mathbf{x}(k)}, k=0,1, \ldots$,

where $\mu>0$ denotes a small step size, $\mathbf{T}(0)$ is the initial position of the sound source. The iterations are stopped when the gradient term is sufficiently close to zero.

The Cramér-Rao lower bound (CRLB), which is equal to the inverse of the Fisher information matrix (FIM), provides a performance lower bound for the asymptotically unbiased estimator [30,31]. The FIM for MLE can be given by [30]

$\mathbf{F}=\left[\begin{array}{cc}\sum_{k=1}^{N} \frac{\cos ^{2} \theta_{k}}{r_{k}^{2} \sigma_{k}^{2}} & -\frac{1}{2} \sum_{k=1}^{N} \frac{\sin 2 \theta_{k}}{r_{k}^{2} \sigma_{k}^{2}} \\ -\frac{1}{2} \sum_{k=1}^{N} \frac{\sin 2 \theta_{k}}{r_{k}^{2} \sigma_{k}^{2}} & \sum_{k=1}^{N} \frac{\sin ^{2} \theta_{k}}{r_{k}^{2} \sigma_{k}^{2}}\end{array}\right]$.

The determinant of FIM can be obtained by

$\operatorname{det}(\mathbf{F})=\frac{1}{4}\left\{\left(\sum_{k=1}^{N} \frac{1}{r_{k}^{2} \sigma_{k}^{2}}\right)^{2}-\left(\sum_{k=1}^{N} \frac{\cos 2 \theta_{k}}{r_{k}^{2} \sigma_{k}^{2}}\right)^{2}-\left(\sum_{k=1}^{N} \frac{\sin 2 \theta_{k}}{r_{k}^{2} \sigma_{k}^{2}}\right)^{2}\right\}$,

$\operatorname{det}(\mathbf{F})=\sum_{S} \frac{\sin ^{2}\left(\theta_{i}-\theta_{j}\right)}{r_{i}^{2} r_{j}^{2} \sigma_{k}^{2} \sigma_{k}^{2}}, j>i$.

$S=\{(i, j)\}$ is the set of all combinations of $i$ and $j$, with $i, j \in$ $\{1,2, \ldots, N\}$ and $j>i$. From (12), we can see that the FIM determinant is approximately inversely proportional to the variance of the 


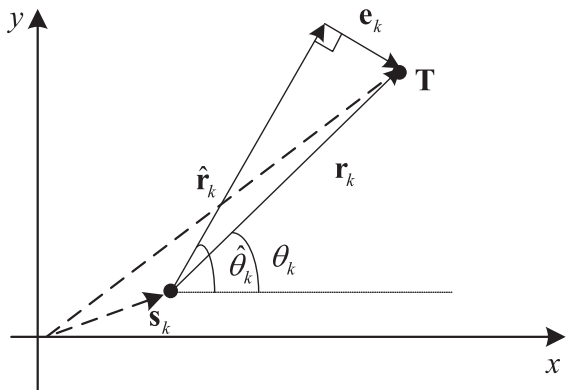

Fig. 2. The orthogonal relationship between the measured and true bearing vector.

bearing measurement errors for a given sensor placement; as a result, the FIM determinant will decrease when one or more variances of bearing measurement errors become larger because of the uncertainty.

Here we use a three- sensors localization system as an example to illustrate the effect of the outlier problem. The three sensors are evenly deployed on a circle with a radius of $1 \mathrm{~m}$, and the source is located in the centre, i.e. $r_{1}=r_{2}=r_{3}=1, \theta_{1}=0^{\circ}, \theta_{2}=120^{\circ}$, and $\theta_{3}=-120^{\circ}$; Under this assumption, (12) can be rewritten as

$\operatorname{det}(\mathbf{F})=\frac{3}{4}\left(\frac{1}{\sigma_{1}^{2} \sigma_{2}^{2}}+\frac{1}{\sigma_{1}^{2} \sigma_{3}^{2}}+\frac{1}{\sigma_{2}^{2} \sigma_{3}^{2}}\right)$.

Normally, $\sigma_{1}=\sigma_{2}=\sigma_{3}=\sigma$ should be small enough, e.g., $\sigma=$ $1^{\circ}$, to ensure accurate localization. However, if one of the sensors fails, then one of the $\sigma$ will be much larger, for example, $\sigma_{2}=\sigma_{3}=$ $1^{\circ}$ and $\sigma_{1}=20^{\circ}$. The corresponding values of (13) are $2.42 \times 10^{7}$ and $8.12 \times 10^{6}$. Thus, an outlier may decrease FIM significantly.

\subsection{Pseudolinear estimator and its outlier effect}

In reference [5], a pseudolinear estimator (PLE), known as the orthogonal vectors (OV) method for source localization, was proposed based on the orthogonal vector relationship between the bearing measurement vectors of AOA with and without noise, denoted as $\hat{\mathbf{r}}_{k}$ and $\mathbf{r}_{k}$, respectively. $\mathbf{e}_{k}$ is the orthogonal error vector, as shown in Fig. 2. The geometric relationship can be expressed as follows:

$\mathbf{e}_{k}=r_{k} \sin \left(\hat{\theta}_{k}-\theta_{k}\right)\left[\begin{array}{c}\sin \hat{\theta}_{k} \\ -\cos \hat{\theta}_{k}\end{array}\right]$,

where $r_{k}=\left\|\mathbf{r}_{k}\right\|$ is the distance from $\mathbf{s}_{k}$ to the target. From the geometric relationship, we can obtain

$\mathbf{T}=\mathbf{s}_{k}+\mathbf{r}_{k}=\mathbf{s}_{k}+\hat{\mathbf{r}}_{k}+\mathbf{e}_{k}$.

Using the transpose of orthogonal unit vector $\mathbf{a}_{k}=$ $\left[\sin \hat{\theta}_{k}-\cos \hat{\theta}_{k}\right]^{\mathrm{T}}$ and multiplying it by $(15)$ yields

$\mathbf{a}_{k}^{\mathrm{T}} \mathbf{T}=\mathbf{a}_{k}^{\mathrm{T}} \mathbf{s}_{k}+\mathbf{a}_{k}^{\mathrm{T}} \mathbf{e}_{k}$.

The matrix form can be rewritten as

$\mathbf{A T}=\mathbf{B}+\mathbf{e}$,

where

$$
\begin{aligned}
& \mathbf{A}=\left[\begin{array}{cc}
\sin \hat{\theta}_{1} & -\cos \hat{\theta}_{1} \\
\sin \hat{\theta}_{2} & -\cos \hat{\theta}_{2} \\
\vdots & \vdots \\
\sin \hat{\theta}_{N} & -\cos \hat{\theta}_{N}
\end{array}\right], \quad \text { and } \quad \mathbf{B}=\left[\begin{array}{c}
x_{1} \sin \hat{\theta}_{1}-y_{1} \cos \hat{\theta}_{1} \\
x_{2} \sin \hat{\theta}_{2}-y_{2} \cos \hat{\theta}_{2} \\
\vdots \\
x_{N} \sin \hat{\theta}_{N}-y_{N} \cos \hat{\theta}_{N}
\end{array}\right] \\
& \mathbf{e}=\left[\begin{array}{llll}
r_{1} \sin \varepsilon_{1} & r_{2} \sin \varepsilon_{2} & \ldots & r_{N} \sin \varepsilon_{N}
\end{array}\right]^{\mathrm{T}}, \\
& \varepsilon_{k}=\hat{\theta}_{k}-\theta_{k} .
\end{aligned}
$$

The estimated position of the target is given by

$$
\hat{\mathbf{T}}_{P L E}=\left(\mathbf{A}^{\mathrm{T}} \mathbf{A}\right)^{-\mathbf{1}} \mathbf{A}^{\mathrm{T}} \mathbf{B} \text {. }
$$

If the measurement error is small enough, we can have $\sin \varepsilon_{k} \approx$ $\varepsilon_{k}$, then the approximation of the residuals can be expressed as $e_{k} \approx r_{k} \varepsilon_{k}$. The position error is

$$
\begin{aligned}
\triangle \mathbf{T}_{P L E} & =\hat{\mathbf{T}}_{P L E}-\mathbf{T} \\
& =\left(\mathbf{A}^{\mathbf{T}} \mathbf{A}\right)^{-\mathbf{1}} \mathbf{A}^{\mathrm{T}} \mathbf{B}-\left(\mathbf{A}^{\mathbf{T}} \mathbf{A}\right)^{-\mathbf{1}} \mathbf{A}^{\mathbf{T}} \mathbf{A} \mathbf{T} \\
& =\left(\mathbf{A}^{\mathbf{T}} \mathbf{A}\right)^{-\mathbf{1}} \mathbf{A}^{\mathbf{T}}(-\mathbf{e}) .
\end{aligned}
$$

The covariance matrix of estimation error can be formulated as

$$
\operatorname{cov}\left(\Delta \mathbf{T}_{P L E}\right)=E\left(\Delta \mathbf{T}_{P L E} \Delta \mathbf{T}_{P L E}^{\mathrm{T}}\right) .
$$

The MSE (mean-square error) is given by

$$
M S E_{P L E}=\operatorname{tr}\left(\operatorname{cov}\left(\Delta \mathbf{T}_{P L E}\right)\right),
$$

$$
\begin{aligned}
M S E_{P L E}= & \frac{1}{\sum_{i, j \in S} \sin ^{2}\left(\theta_{i}-\theta_{j}\right)} \sum_{i=1}^{N} \\
& \times\left\{\left\{\sigma_{e_{i}}^{2}\left[\left(f_{11} \sin \theta_{i}-f_{12} \cos \theta_{i}\right)^{2}+\left(f_{21} \sin \theta_{i}-f_{22} \cos \theta_{i}\right)^{2}\right]\right\},\right.
\end{aligned}
$$

where $f_{11}=\sum_{i=1}^{N} \sin ^{2} \theta_{i}, \quad f_{22}=\sum_{i=1}^{N} \cos ^{2} \theta_{i}, \quad$ and $\quad f_{21}=f_{12}=$ $\sum_{i=1}^{N} \cos \theta_{i} \sin \theta_{i}$,

$\sigma_{e_{i}}^{2}=E\left(\mathbf{e e}^{\mathrm{T}}\right)$.

Because $\sin ^{2} \varepsilon_{i}$ is very small, we can have $\sigma_{e_{i}}^{2} \approx r_{i}^{2} \sigma_{i}^{2}$ from (19) and (25).

We can see from (24) that MSE is affected by the relative geometry between the target and the sensors, the number of sensors and the error of measurements. Now we only address the effect of outlier bearing measurements. Same to MLE when $r_{1}=r_{2}=r_{3}=1$, $\theta_{1}=0^{\circ}, \theta_{2}=120^{\circ}$, and $\theta_{3}=-120^{\circ}$, we set $\sigma_{1}=\sigma_{2}=\sigma_{3}=1^{\circ}$ and $\sigma_{2}=\sigma_{3}=1^{\circ}, \sigma_{1}=20^{\circ}$ for the cases of 1) all the sensors being good and 2) one sensor failing, respectively. The MSEs of PLE are $9.14 \times 10^{-4}$ and 0.1225 for case 1 and case 2 , respectively. It is clear that the outlier caused by uncertainty has a great influence on the localization error.

\section{The robust localization method with uncertain noise model}

\subsection{Bearing measurement error model}

As mentioned above, when a sensor network used in practical application, the measured data is not always reliable. The limited capability and resource of low quality and low cost sensor nodes make the unreliable data present. The probability of unreliable data will grow rapidly especially when battery power is exhausted [32]. On the other hand, the harsh and unattended environments may affect sensors, resulting in unexpected noisy interference or missing data. In the application considered in this work, the above factors will lead to unreliability of the bearing measurements. For these uncertain environments, the estimated bearings can be any direction within the range of $[-\pi, \pi)$. Under this situation, we assume that the estimated bearings are contaminated by a uniform distribution with probability, which can be modelled as a mixture model of Gaussian distribution and uniform distribution

$F=\left(1-p_{i}\right) N\left(\theta_{i}, \sigma_{i}^{2}\right)+p_{i} U(-\pi, \pi)$

where $\mathrm{F}$ denoted the probability density function (pdf) of $\hat{\theta}_{i} . p_{i}$ is the outlier occurrence probability, $N\left(\theta_{i}, \sigma_{i}^{2}\right)$ denotes the pdf of 
Gaussian distribution with mean $\theta_{i}$ and variance $\sigma_{i}^{2} . U(-\pi, \pi)$ is the uniform pdf under the interval of $[-\pi, \pi)$. The mixture model belongs to a heavy-tail distribution class [18, 22]; and it models how the Gaussian component mixing with the non-Gaussian noise portion of the distribution. Therefore, it is reasonable to model the impulsive outliers occurring in an uncertain environment.

\subsection{M-estimator and iterative reweighted nonlinear least squares method}

\subsubsection{M-estimator}

An M-estimator proposed by Huber [33] can be seen as a generalized MLE that aims to minimize the robust cost functions. An important property of a robust cost function $\rho$ is that it is immune for the undesired data during the estimation and yields comparable performance as the traditional MLE. Assuming that the derivative of $\rho$ is denoted as $\psi$, i.e., $\psi(a)=\rho^{\prime}(a)$, a cost function $\rho$ is convergent if and only if the following conditions are satisfied [34]:

(1) $\rho(a)$ is continuous, and $\rho(0)=0$.

(2) $\rho(a)$ is monotonically increasing on $[0, \infty)$.

(3) $\frac{\psi(a)}{a}$ decreases monotonically and is bounded above on $(0, \infty)$.

A weight function $\omega$ is defined as

$\omega(a)=\left\{\begin{array}{cc}\frac{\psi(a)}{a}, & a \neq 0 \\ \psi^{\prime}(0), & a=0\end{array}\right.$

The common used robust cost functions are Huber's function $\rho_{\mathrm{Hu}}$, Cauchy's function $\rho_{\mathrm{Ca}}$, and Tukey's bi-weight function $\rho_{\mathrm{Tu}}[30]$ :

Huber's function:

$\rho_{H u}(a)=\left\{\begin{array}{ll}\frac{1}{2} a^{2} & \text { if }|a| \leq k_{H u} \\ k_{H u}|a|-\frac{1}{2} k_{H u}^{2} & \text { if }|a|>k_{H u}\end{array}\right.$,

$\omega_{H u}(a)=\left\{\begin{array}{cc}1 & \text { if }|a| \leq k_{H u} \\ \frac{k_{H u}}{|a|} & \text { if }|a|>k_{H u}\end{array}\right.$,

Cauchy's function:

$\rho_{C a}(a)=\frac{k_{C a}^{2}}{2} \log \left(1+\left(\frac{a}{k_{C a}}\right)^{2}\right)$,

$\omega_{C a}(a)=1 /\left(1+\left(\frac{a}{k_{C a}}\right)^{2}\right)$,

Tukey's bi-weight function:

$\rho_{T u}(a)= \begin{cases}\frac{k_{T u}^{2}}{6}\left\{1-\left(1-\left(\frac{a}{k_{T u}}\right)^{2}\right)^{3}\right\} & \text { if }|a| \leq k_{T u}, \\ \frac{k_{T u}^{2}}{6} & \text { if }|a|>k_{T u}\end{cases}$

$\omega_{T u}(a)= \begin{cases}\left(1-\left(\frac{a}{k_{T u}}\right)^{2}\right)^{2} & \text { if }|a| \leq k_{T u}, \\ 0 & \text { if }|a|>k_{T u}\end{cases}$

where $k_{\mathrm{Hu}}$ and $k_{\mathrm{Tu}}$ are bound parameters. To get a more straightforward sense, the least squares (LS) function and these three robust cost functions are compared in Fig. 3 with $k_{\mathrm{Hu}}=0.6, k_{\mathrm{Ca}}=0.9$ and $k_{T \mathrm{u}}=0.9$. Note that traditional LS estimator is more likely to be dominated by large estimated residuals. Alternatively, the three robust cost functions exhibit more robust performance by discounting the outliers. Tukey's bi-weight function has the strongest ability to against outliers, followed by Cauchy function. Huber's cost function is a very conservative algorithm that is based on decreasing the cost value for large residuals. However, it is still robust and, more importantly, it is convex; i.e., it does not introduce local minima to some extent. In the following sections, we will focus on Tukey's bi-weight function and Huber's function.

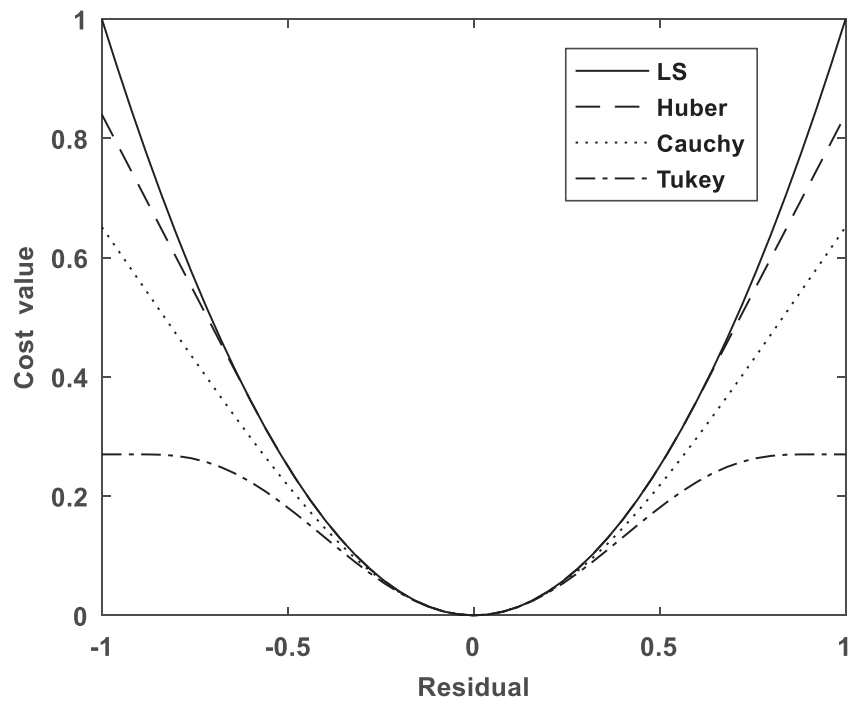

Fig. 3. Different cost functions via residuals when $k_{H u}=0.6, k_{C a}=0.9$ and $k_{T u}=$ 0.9 .

3.2.2. Iterative reweighted nonlinear least squares (IRNLS) method

For an AOA-based localization system, the optimization function based on the M-estimator can be expressed as

$$
\text { minimize } \sum_{i=1}^{N} \rho\left(\frac{\hat{\theta}_{i}-\theta_{i}(\mathbf{T})}{\hat{\sigma}_{i}}\right),
$$

where $\hat{\sigma}_{k}$ denotes the scaling parameter which will be discussed in next section. Setting $\nabla_{\mathbf{x}} \rho=0$, one can get

$$
\begin{aligned}
& \sum_{i=1}^{N} \nabla_{\mathbf{x}} \rho\left(\frac{\hat{\theta}_{i}-\theta_{i}(\mathbf{T})}{\hat{\sigma}_{i}}\right) \\
& =\sum_{i=1}^{N} \omega\left(\frac{\hat{\theta}_{i}-\theta_{i}(\mathbf{T})}{\hat{\sigma}_{i}}\right) \cdot \frac{\hat{\theta}_{i}-\theta_{i}(\mathbf{T})}{\hat{\sigma}_{i}} \cdot \nabla_{\mathbf{x}}\left(\frac{\hat{\theta}_{i}-\theta_{i}(\mathbf{T})}{\hat{\sigma}_{i}}\right)=0
\end{aligned}
$$

If one ignores the dependence of $\mathbf{T}$ by $\omega\left(\frac{\hat{\theta}_{i}-\theta_{i}(\mathbf{T})}{\hat{\sigma}_{i}}\right)$, the above expression (35) can be formulated as the gradient of a weighted least square cost function:

$$
\begin{aligned}
& \nabla_{\mathbf{x}}\left(\sum_{i=1}^{N} \omega_{i}\left(\frac{\hat{\theta}_{i}-\theta_{i}(\mathbf{T})}{\hat{\sigma}_{i}}\right) \cdot\left(\frac{\hat{\theta}_{i}-\theta_{i}(\mathbf{T})}{\hat{\sigma}_{i}}\right)^{2}\right) \\
& \approx 2 \sum_{i=1}^{N} \omega\left(\frac{\hat{\theta}_{i}-\theta_{i}(\mathbf{T})}{\hat{\sigma}_{i}}\right) \cdot \frac{\hat{\theta}_{i}-\theta_{i}(\mathbf{T})}{\hat{\sigma}_{i}} \cdot \nabla_{\mathbf{x}}\left(\frac{\hat{\theta}_{i}-\theta_{i}(\mathbf{T})}{\hat{\sigma}_{i}}\right)
\end{aligned}
$$

As $\hat{\sigma}_{i}$ is a scale parameter which can be neglected, (36) may be solved from an approximated cost function:

$$
\operatorname{minimize} \sum_{i=1}^{N} \omega_{i}\left(\frac{\hat{\theta}_{i}-\theta_{i}(\mathbf{T})}{\hat{\sigma}_{i}}\right)\left(\hat{\theta}_{i}-\theta_{i}(\mathbf{T})\right)^{2} .
$$

An iterative quasi-Newton method [35] can be applied to solve the problem. Specifically, the estimated position in the $m$-th iteration is denoted as

$\mathbf{T}_{m}=\mathbf{T}_{m-1}+\Delta_{m}$

where $\Delta_{m}$ is calculated by solving the followed equation:

$\mathbf{J}_{m}^{\mathrm{T}} \boldsymbol{\omega} \mathbf{J}_{m} \cdot \Delta_{m}=\mathbf{J}_{m}^{\mathrm{T}} \boldsymbol{\omega}(\boldsymbol{\theta}-\hat{\boldsymbol{\theta}})$. 
Table 1

Iterative procedure for IRNLS.

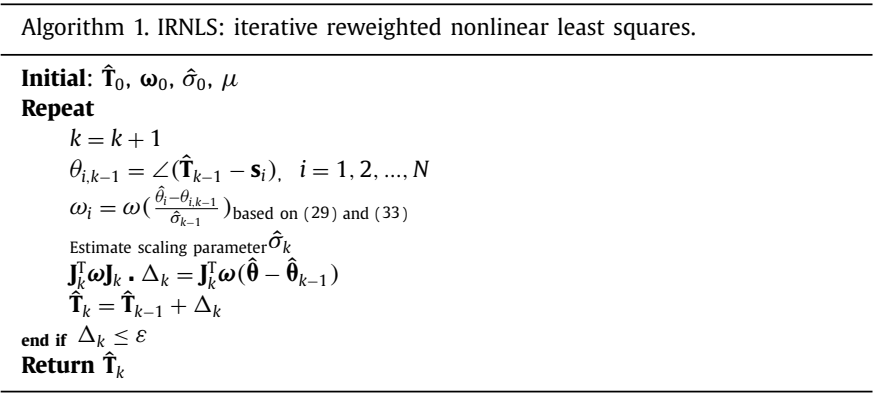

The $i$-th row block of $\mathbf{J}_{\mathrm{m}}$ is

$$
\left[\mathbf{J}_{m}\right]_{i}=\left.\frac{\partial \theta(\mathbf{T})}{\partial \mathbf{T}}\right|_{\mathbf{T}=\hat{\mathbf{T}}_{m-1}}=\frac{1}{\left\|\hat{\mathbf{T}}_{m-1}-\mathbf{s}_{i}\right\|}\left[-\sin \hat{\theta}_{i, m-1} \quad \cos \hat{\theta}_{i, m-1}\right] .
$$

The weight matrix $\boldsymbol{\omega}=\operatorname{diag}\left(\omega_{1}, \ldots, \omega_{N}\right)$ is estimated based on the updated position matrix $\mathbf{T}$.

Until now, the iterative procedure can be summarized as Table 1.

3.2.2.1. Scaling parameter determination approach. For the optimization problem of (37), a scaling parameter $\hat{\sigma}$ should be estimated during an iterative procedure. When $\hat{\sigma}$ is small and $\psi$ approaches zero for large residuals, the cost function will descent slowly near to zero. This feature can decrease the convergence speed or make the function convergence to a local extremum. A method to avoid this problem is to choose a large value for $\hat{\sigma}$, greater than $\sigma^{*}$ (the expected standard deviation of the error of the reliable measurements) in the first iteration, and then gradually approach $\sigma^{*}$ [19]. The iterative descent (ID) method can be formulated as

$\hat{\sigma}_{k}=\left\{\begin{array}{ll}1.90 \operatorname{med}_{i}\left(\left|r_{\theta_{i}}\right|\right) & k=0 \\ \mu\left(\hat{\sigma}_{k-1}-\sigma^{*}\right)+\sigma^{*} & k>0\end{array}\right.$,

where $\mu$ is a parameter satisfying $0 \leq \mu<1, k$ is the iterative number. $r_{\theta_{i}}$ is the bearing residual. $\operatorname{med}_{i}$ denotes the median function, which provides less vulnerability to outliers.

An alternative method which is based on the Gaussian approximation (GAP) to determine the scaling parameter can be given by

$\hat{\sigma}_{k}=1.481 \operatorname{med}_{i}\left(\left|r_{\theta_{i}}-\operatorname{med}\left(r_{\theta_{i}}\right)\right|\right)$,

(the factor 1.481 makes scale parameter as an approximately unbiased estimation when the error model is Gaussian). Here IRNLS_ID and IRNLS_GAP are defined for IRNLS based method when scale parameter is estimated by (41) and (42). TIRNLS_ID and TIRNLS_GAP represent Tukey's cost function solved by IRNLS_ID and IRNLS_GAP, respectively. Similarly, HIRNLS_ID and HIRNLS_GAP denote IRNLS base d method for Huber's cost function. To compare the performance of (41) and (42), 1000 Monte Carlo experiments were conducted when 20 sensors are randomly deployed in a square with length $100 \mathrm{~m}$, and $\sigma=1^{\circ}$.

The statistical results of the localization error in different ranges are shown in Fig. 4. One can see that HIRNLS_ID and HIRNLS_GAP show almost the same performance for both scaling parameters. The percentage of position errors less than $0.5 \mathrm{~m}$ for TIRNLS_GAP is slightly higher than TIRNLS_ID. We can say that (42) exhibits a slightly better performance than (41), while both scaling parameter functions can work well. We will chose (42) to determine the scaling parameter for the remainder of this work. To simplicity, TIRNLS_GAP and HIRNLS_GAP will be replaced by TIRNLS and HIRNLS in the next sections.

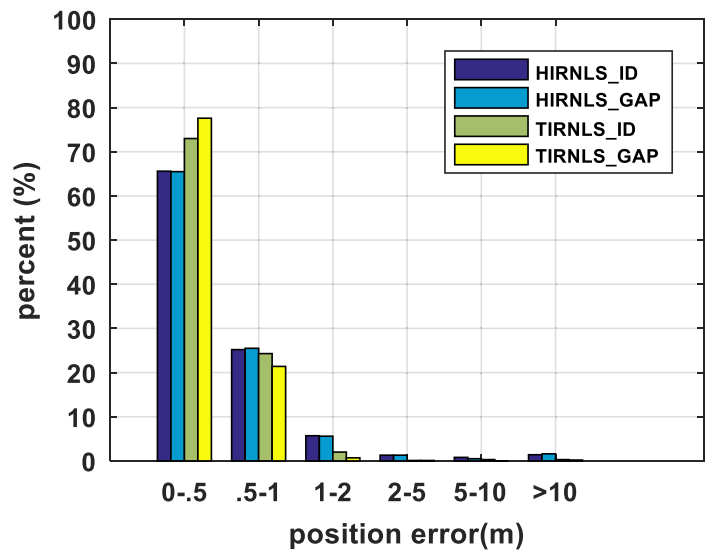

Fig. 4. Histogram of the localization error distribution.

Table 2

Robust estimator based on PLE.

\begin{tabular}{l}
\hline Algorithm 2: Huber's function based on PLE: HPLE. \\
\hline cvx_begin \\
$\quad$ variable $\mathbf{T}(2)$ \\
$\quad$ minimize $\left(\right.$ sum $\left(\right.$ huber $\left.\left(\mathbf{A T}-\mathbf{B}, k_{\mathrm{Hu}}\right)\right)$ ) \\
subject to $l_{1} \leq \mathbf{T} \leq l_{2}$ \\
cvx_end
\end{tabular}

Note: $l_{1}$ and $l_{2}$ are the bound values of the test area

\subsubsection{The robust estimate method based on PLE}

As mentioned before, the IRNLS method is an approximated weighted maximum likelihood estimator. As PLE is simple to implement with low complexity, we will introduce the M-estimator to PLE. Because of the convex property of Huber's cost function, the optimization problem can be transformed into second-order cone program (SOCP) problem, which can easily be solved by the CVX tool [36]. Moreover, we can add constraints on the optimization procedure. The program is shown in Table 2.

\section{Numerical experiments}

In this section, we will use the simulation result to find the proper bound parameter for different outlier probability. Then, the performances of the proposed methods is compared with MLE, PLE, EM based method [18] by simulations.

\subsection{The relationship between bound parameter $\mathrm{k}$ and outlier probability}

As shown in Fig. 3, the performance of an M-estimator depends on the bound parameter. A fixed bound value which can be determined by 3 -sigma rule and confidence level rule [33] is usually used in commonly methods. To guarantee the robustness of the M-estimator, the bound parameter $k$ can be chosen in the way where the asymptotic variance $v$ is close to 1 with the assumption $a \sim N(0,1)$.

$v=\frac{E\left[\psi^{2}(a)\right]}{E^{2}\left[\psi^{\prime}(a)\right]}$

The asymptotic variance $v$ should be greater than 1 for robust cost functions working well when there is no outlier. By choosing it to be 1.01, the bound parameters for Huber and Tukey functions are $k_{H u}=2.01$ and $k_{T u}=7.05$, respectively [19]. However, the solution is not optimal when outliers are present for $k_{H u}=2.01$ and $k_{T u}=7.05$.

In this section, simulation experiments are conducted to investigate the relationship between bound parameters and outlier 


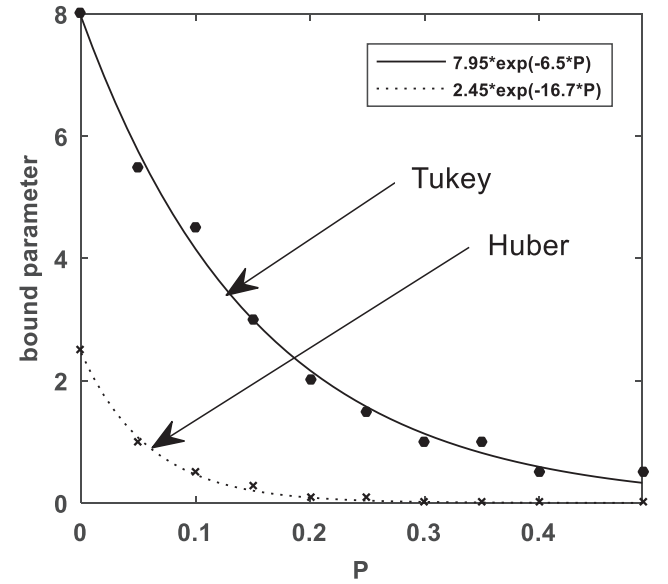

Fig. 5. The bound parameter via outlier probability when $\mathrm{N}=10, \sigma=1^{\circ}$.

probability $p_{i}$. Assume that 10 sensors are randomly deployed in a $100 \mathrm{~m} \times 100 \mathrm{~m}$ test field. To better compare the localization performance for different bound parameters, each sensor is assumed to have the same outlier probability, i.e., $p_{s_{1}}=p_{s_{2}}=\ldots=p_{s_{N}}=p$. The standard deviation of Gaussian component is $\sigma=1^{\circ}$. When $p$ varies from 0 to 0.49 , the root mean square error (RMSE) of 5000 trials is conducted to compare the localization performance.

Fig. 5 shows that the approximate optimal bound parameter that gives the smallest RMSE declines with the outlier probability increasing. That is to say, when outlier probability increases, the M estimator needs to be more aggressive in discounting larger value of residuals. From the simulation results, we can observe that the relationship between the approximate optimal bound parameter and outlier probability fits well with exponential functions which can be written as,

$k_{T u}=7.95 e^{-6.5 P}$

$k_{H u}=2.45 e^{-16.7 P}$

The Potential influence of the standard deviation $\sigma$ on bound parameters selection is also investigated for both Huber's function and Tukey's function. Simulation results are shown in Fig. 6 when $p=0.15$. From these results, we can see that $\sigma$ has little impact on the selection of bound parameters.

\subsection{Simulation results}

In this section, the simulations are conducted to verify the effectiveness and robustness for the proposed methods, TIRNLS, HIRNLS and HPLE, by comparing them with PLE, MLE and EM based method [18]. Assume that $N$ sensors are randomly deployed in a $100 \mathrm{~m} \times 100 \mathrm{~m}$ test area. Every sensor is equipped with a microphone array to estimate sound source AOAs. The RMSE of 1000 trials is calculated as the performance metric. For every simulation, the localization result of PLE is used as the initial position for TIRNLS, HIRNLS, MLE and EM based methods. The maximum number of iterations for MLE, IRNLS and EM based method is set to be 50.The iterative procedure is aborted when either the maximum number of iterations is exceeded or when the distance between the estimated positions in two successive iterations is less than $10^{-5} \mathrm{~m}$. In this section, the outlier probability is assumed to be the same for all the sensors, $p_{1}=p_{2}=\ldots=p$. The bound parameters are chosen based on (44) and (45).

We set the acoustic source position to be located at $[65 \mathrm{~m}$, $50 \mathrm{~m}]^{\mathrm{T}}$, the estimation bias and RMSE of the estimated positions obtained from 1000 Monte Carlo trials for different outlier probability are plotted in Fig. 7 and Fig. 8. The standard deviation of the bearing estimation errors is $\sigma=1^{\circ}$. The number of sensors is $N=10$. To avoid the influence of non-convergent results on the RMSE for MLE, we set the tolerant position error to be $50 \mathrm{~m}$.

It can be seen in Fig. 7 that the PLE show the highest bias among all the methods when the outlier probability is less than 0.4 . The ML estimator, which is an asymptotically unbiased under Gaussian noise, also exhibits very high bias and even goes beyond the PLE when the outlier probability is greater o.4. The EM algorithm which is based on the Gaussian component identification has much smaller bias compared with PLE and MLE. Among the three proposed methods, the bias of HIRNLS is the largest but still significantly smaller than that of PLE and MLE. The bias of HPLE and TIRNLS are almost the same.

The RMSE results also show that the localization performances of PLE and MLE are easily affected by outliers. And the MLE may converge to local optimum with great chance when the initial position is far away from the true value. The RMSEs of the three proposed methods and EM based method also increase with the outlier probability, but with a significant improvement compared with MLE and PLE. HPLE has the lowest localization error; this is because it is based on convex optimization, which can prevent the optimization function falling into a local optimum [37]. We can

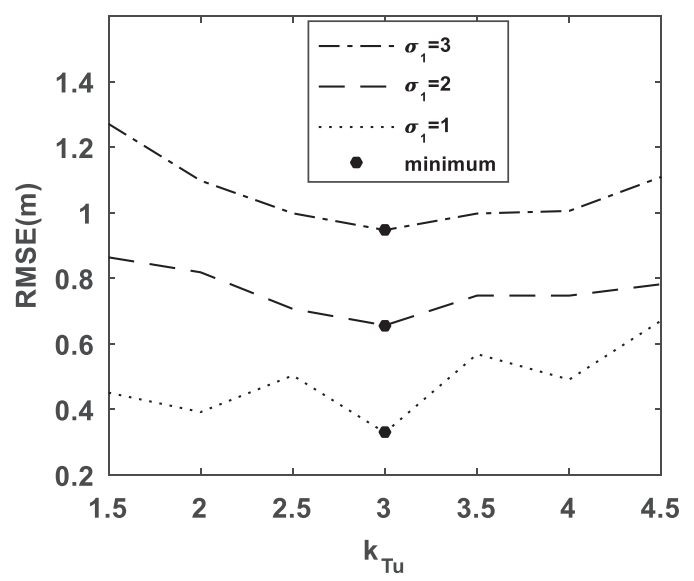

(a)

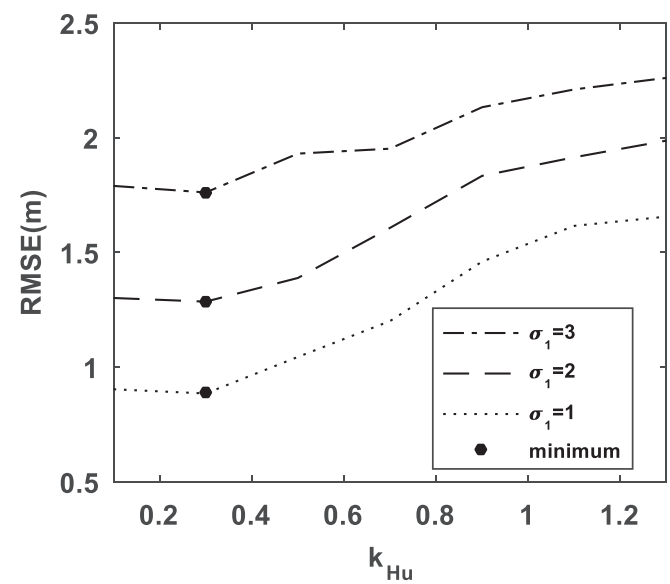

(b)

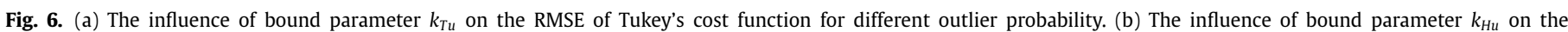
RMSE of Huber's cost function for different $\sigma$. 


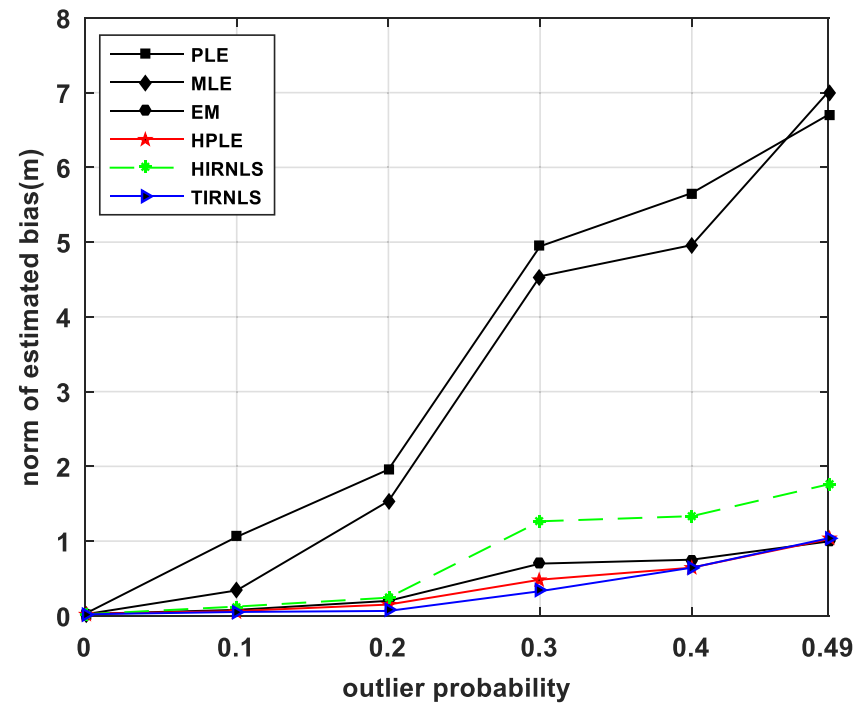

Fig. 7. Estimation bias at different outlier probability for PLE, MLE, EM, HIRNLS, TIRNLS and HPLE $\left(\sigma=1^{\circ}, \mathrm{N}=10\right)$.

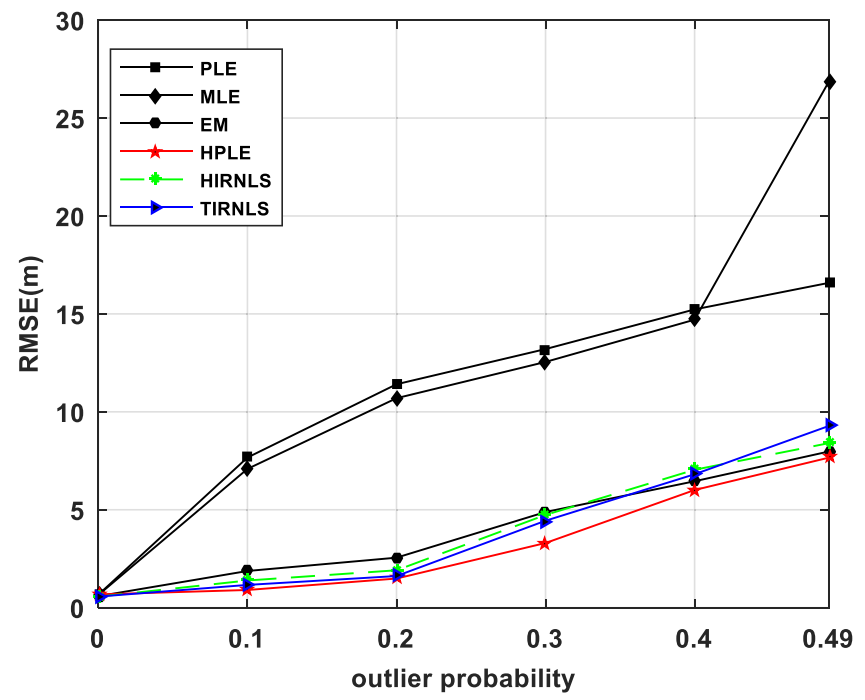

Fig. 8. RMSE vs different outlier probabilities for PLE, MLE, EM, HIRNLS, TIRNLS and HPLE $\left(\sigma=1^{\circ}, \mathrm{N}=10\right)$.

also observe that the RMSEs of TIRNLS and HIRNLS are lower than that of EM based method when outlier probability $p$ is less than 0.3. For larger outlier probability, EM exhibits a slightly better performance than TIRNLS and HIRNLS but still performs poorer than HPLE. Besides, as the Tukey's function is more aggressive than Huber's function in against outliers as shown in Fig. 3, the localization results of HIRNLS exhibit more conservative than TIRNLS.

As mentioned in Section 2.2 and Section 2.3, the localization performance is also affected by the standard deviation of the bearing estimated error. To illustrate this, we simulated the estimation bias and RMSE of different algorithms at different $\sigma$ when $N=10$ and $p=0.2$. Fig. 9 and Fig. 10 show the simulation results of 1000 trials.

From Fig. 9, we can observe that the estimation bias of the proposed methods seem to be insensitive to the bearing estimated error compared with PLE and MLE. The RMSEs become large for all the methods as $\sigma$ increases. In addition, the localization accuracy of the three proposed localization method shows their robustness against outliers as $\sigma$ increases, especially for HPLE and TIRNLS, both of which exhibit lower RMSEs than EM based method. As $\sigma$

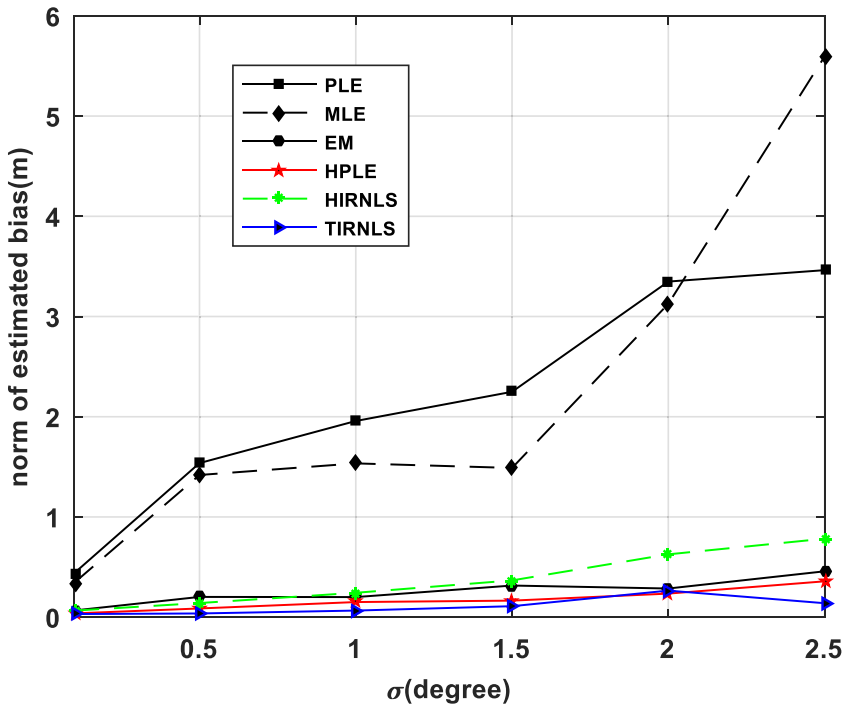

Fig. 9. Estimation bias vs. different $\sigma$ for PLE, MLE, EM and HIRNLS, TIRNLS and $\operatorname{HPLE}(N=10$ and $p=0.2)$.

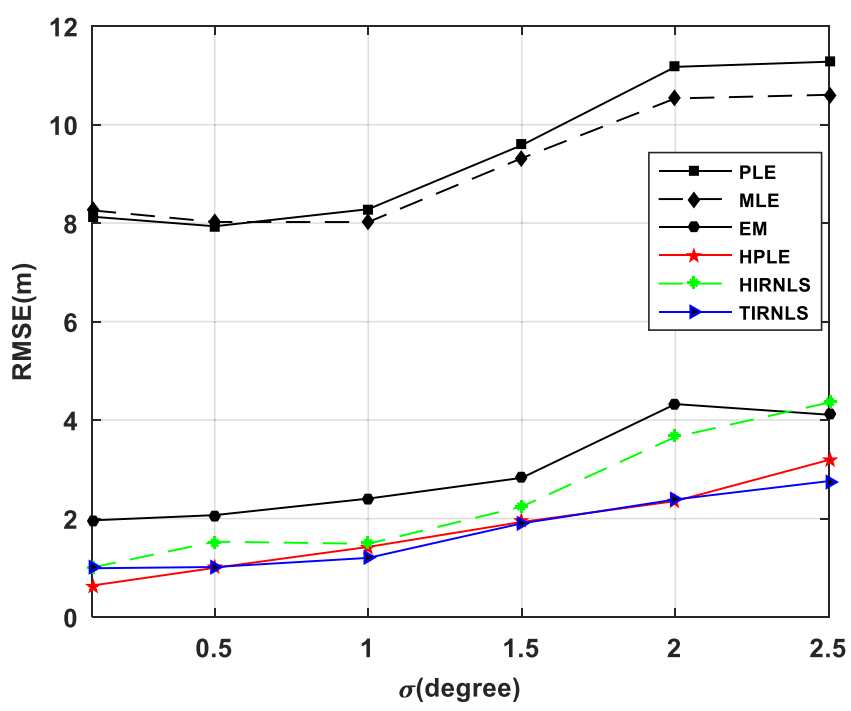

Fig. 10. RMSE for different $\sigma$ for PLE, MLE, EM, HIRNLS, TIRNLS and HPLE ( $N=10$ and $p=0.2$ ).

increases, the conservative HIRNLS method becomes more difficult to identify the outliers. The difference of RMSEs between HIRNLS and EM based methods becomes smaller as $\sigma$ increases.

An important property of the M-estimator is convergence. Fig 11 plots the convergence performances of TIRNLS and HIRNLS when the outlier probability is set to be 0 and 0.2 , respectively. As we can see from the results, both TIRNLS and HIRNLS methods have a fast convergence speed. Fig. 11(b) displays the estimated error distribution for 1000 trials when $p=0.2$, clearly showing that more than $90 \%$ of the errors are less than $3 \mathrm{~m}$ for the three proposed methods, and nearly $88 \%$ for EM based method while only $20 \%$ for traditional methods. Note that the TIRNLS and HIRNLS methods are iterative search methods which are similar with MLE to find the solution, so they may converge to a local optimal. The HPLE, however, does not have this problem.

Fig. 12 shows the simulation results for different number of sensors when the sound source position is different. We can observe that the RMSEs of different localization methods decrease as the number of sensors increases. The three proposed methods exhibit their superiority over EM based method in against outliers 


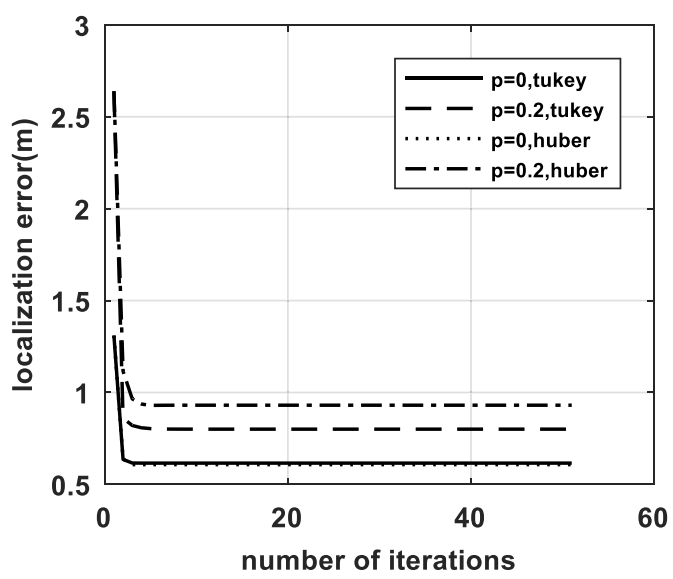

(a) Convergence of IRNLS

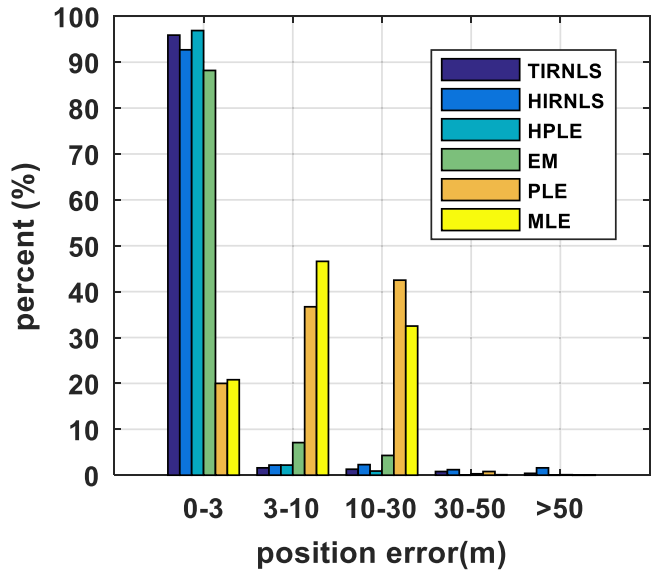

(b) Position error distribution for 1000 trials

Fig. 11. Convergence of the robust estimator and the error distribution of the traditional and proposed methods.

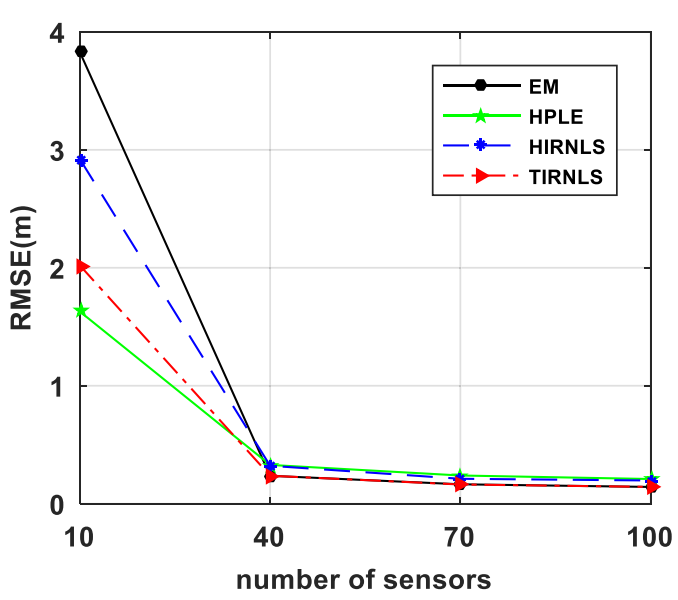

(a). $\sigma=1^{\circ}$ and $p=0.2, \quad \mathbf{T}=[65,50]^{\mathrm{T}} \mathrm{m}$

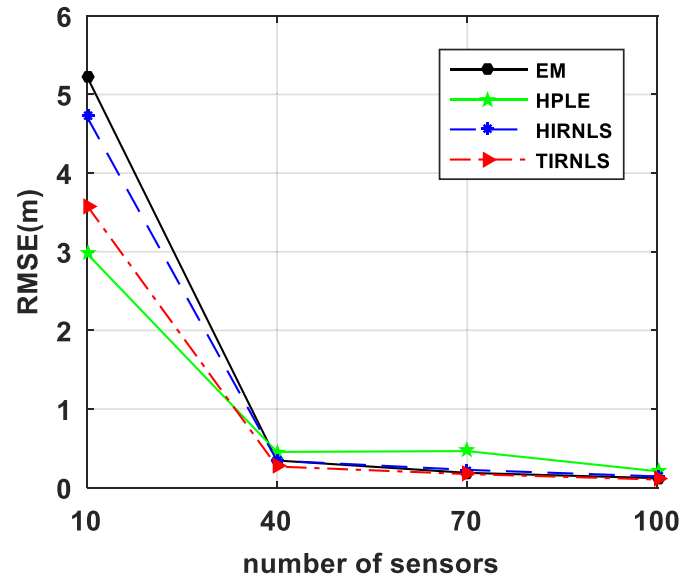

(b). $\sigma=1^{\circ}$ and $p=0.2, \quad \mathbf{T}=[80,80]^{\mathrm{T}} \mathrm{m}$

Fig. 12. The RMSE vs. the number of sensors when $\sigma=1^{\circ}$ and $p=0.2$.

when the number of sensors is small. With the number of sensors increasing, the RMSE of EM based method becomes a little lower than the values of HIRNLS and HPLE methods, and approaches the same value with TIRNLS for the source position $\mathbf{T}=[65,50]^{\mathrm{T}} \mathrm{m}$. Note that, the TIRNLS method always shows a slightly better localization performance with the number of sensors increasing when the sound source located at $[80,80]^{\mathrm{T}} \mathrm{m}$.

To explore the bias performance of the proposed methods as the number of sensors increases, we conduct the bias statistical analysis of the localization results for 1000 simulations. The parameters are set to be the same with Fig 12(a). As we can see from Fig 13, the HIRNLS and TIRNLS are asymptotically unbiased as the number of sensors increases. Even though the HPLE exhibits smaller bias than HIRNLS, the bias does not approach to zero as it is based on the biased estimator PLE.

From Fig. 7 to Fig. 13, we can see that the three proposed methods are robust and efficient for the accurate localization in presence of unreliable measurements. The performance of HPLE and HIRNLS is slightly poorer than TIRNLS. The advantage of HPLE is that it does not require initial value and converges to global optimal as it is based on SOCP. However, it is based on pseudolinear equation which results in the biased performance. In summary, the TIRNLS method performs best among the three proposed methods considering the robustness and the localization accuracy.

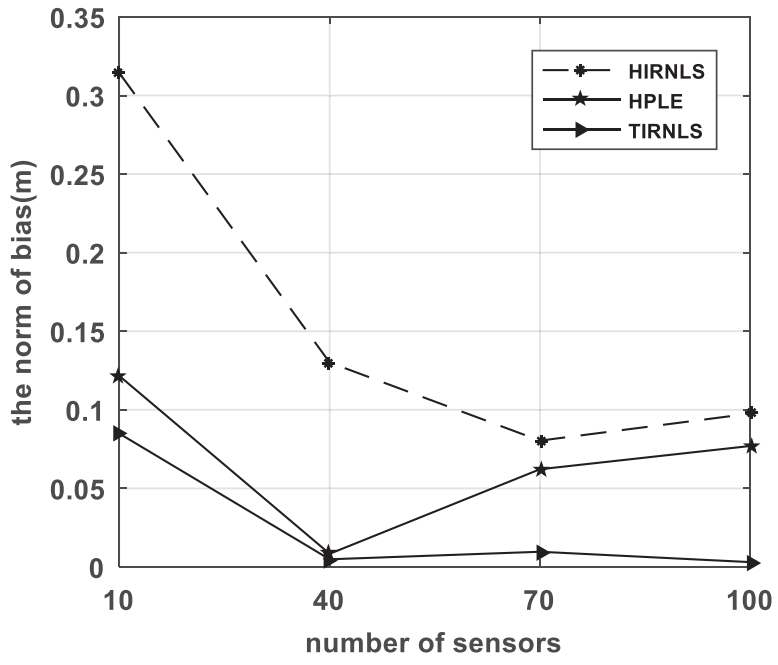

Fig. 13. The bias vs. the number of sensors when $\sigma=1^{\circ}$ and $p=0.2$. 


\section{Conclusions}

The M-estimator, initially designed for the outlier processing, is employed to conduct the AOA based acoustic source localization, and specifically to tackle the performance degradation problem caused by outliers. Three methods, including TIRNLS, HIRNLS and HPLE, are proposed to obtain robust localization performance with the measurement error modelled as a mixture of Gaussian and uniform distribution. The bound parameter in the proposed methods is determined by establishing its relationship with the outlier probability. The localization performances of the proposed methods are compared with EM based method, MLE and PLE. The simulation results illustrate that the proposed methods exhibits much better robustness and accuracy than MLE and PLE. The three proposed methods work better than EM method when a small number of sensors are used. Among the three methods, although comparable to each other, TIRNLS turns out to be the best for different number of sensors.

\section{Acknowledgments}

This work is co-financed by the National Natural Science Foundation of China (No. 61501374), NSFC-Zhejiang Joint Fund for the Integration of Industrialization and Information (U1609204).

\section{References}

[1] G. Han, H. Xu, T.Q. Duong, et al., Localization algorithms of wireless sensor networks: a survey, Telecommun. Syst. 52 (4) (2013) 2419-2436.

[2] P. Transfeld, U. Martens, H. Binder, et al., Acoustic event source localization for surveillance in reverberant environments supported by an event onset detection, in: Proceedings of IEEE International Conference on Acoustics, Speech and Signal Processing (ICASSP), 2015.

[3] A.H. Sayed, A. Tarighat, N. Khajehnouri, Network-based wireless location: challenges faced in developing techniques for accurate wireless location information, IEEE Signal Process. Mag. 22 (4) (2005) 24-40.

[4] R.G. Stansfield, Statistical theory of DF fixing, J. IEE-Part IIIA 94 (15) (1974) $762-770$.

[5] D. Koks, Passive geolocation for multiple receivers with no initial state estimate, Defence Science \& Technology Organization, Edinburgh, SA, Australia, 2001 Technical Report, DSTO RR-0222, November.

[6] K. Dogancay, Bias compensation for the bearings-only pseudolinear target track estimator, IEEE Trans. Signal Process. 54 (1) (2006) 59-68.

[7] J.C. Chen, K. Yao, R.E. Hudson, Acoustic source localization and beamforming: theory and practice, EURASIP J. Adv. Signal Process. 4 (2003) 926-837.

[8] K. Pingarn, Passive position location estimation using the extended Kalman filter, IEEE T. Aero. Elec. Sys. 4 (1987) 558-567.

[9] S.K. Rao, Pseudo-linear estimator for bearings-only passive target tracking, IEEE P-Radar, Son. Nav. 148 (1) (2001) 16-22.

[10] K. Dogancay, Bearings-only target localization using total least squares, Signal Process. 85 (9) (2005) 1695-1710.

[11] W.C. Lee, Uncertainty in wireless sensor networks, Workshop on AFRL, 2010.

[12] N. Patwari, J.N. Ash, S. Kyperountas, et al., Locating the nodes: cooperative localization in wireless sensor networks, IEEE Signal Process. Mag. 22 (4) (2005) 54-69.
[13] P. Rawat, K.D. Singh, H. Chaouchi, et al., Wireless sensor networks: a survey on recent developments and potential synergies, J. Supercomput. 68 (1) (2014) $1-48$.

[14] K. Yu, Y.J. Guo, Statistical NLOS identification based on AOA, TOA, and signal strength, IEEE Trans. Veh. Technol. 58 (1) (2009) 274-286.

[15] K. Ni, G. Pottie, Bayesian selection of non-faulty sensors, in: IEEE International Symposium on Information Theory, 2007, pp. 616-620. (ISIT).

[16] P. Giménez-Febrer, A. Pages-Zamora, S.S. Pereira, et al., Distributed AOA-based source positioning in NLOS with sensor networks, in: IEEE International Conference on Acoustics, Speech and Signal Processing (ICASSP), 2015, pp. 3197-3201.

[17] S.S. Pereira, R. Lòpez-Valcarce, A. Pagès-Zamora, A diffusion-based EM algorithm for distributed estimation in unreliable sensor networks, IEEE Signal Process. Lett. 20 (6) (2013) 595-598.

[18] Q. Yan, J. Chen, G. Ottoy, et al. An accurate AOA localization method based on unreliable sensor detection. IEEE Sens. Appl. Symp., 2018: 1-6.

[19] P. Bergström, O. Edlund, Robust registration of point sets using iteratively reweighted least squares, Comput. Optim. Appl. 58 (3) (2014) 543-561.

[20] P.J. Rousseeuw, A.M. Leroy, Robust Regression and Outlier Detection, Vol. 589, John Wiley \& Sons, 2005

[21] Q. Collier, J. Veraart, B. Jeurissen, et al., Iterative reweighted linear least squares for accurate, fast, and robust estimation of diffusion magnetic resonance parameters, Magn. Reson. Med. 73 (6) (2015) 2174-2184.

[22] Y. Liu, Y.H. Hu, O. Pan, Distributed, robust acoustic source localization in a wireless sensor network, IEEE Trans. Signal Process. 60 (8) (2012) 4350-4359.

[23] J. Bordoy, C. Schindelhauer, R. Zhang, et al., Robust extended Kalman filter for NLOS mitigation and sensor data fusion, IEEE International Symposium on Inertial Sensors and Systems (INERTIAL), 2017.

[24] H. He, J. Chen, J. Benesty, et al., Multichannel time delay estimation for acoustic source localization via robust adaptive blind system identification, in: IEEE International Workshop on Acoustic Signal Enhancement (IWAENC), 2016, pp. 1-5.

[25] D.D. Lee, R.L. Kashyap, Robust maximum likelihood bearing estimation in contaminated Gaussian noise, IEEE Trans. Signal Process. 40 (8) (1992) 1983-1986.

[26] Y. Yardimci, A.E. Cetin, J.A. Cadzow, Robust direction-of-arrival estimation in non-Gaussian noise, IEEE Trans. Signal Process. 46 (5) (1998) 1443-1451.

[27] O. Besson, Y. Abramovich, B. Johnson, Direction-of-arrival estimation in a mixture of K-distributed and Gaussian noise, Signal Process. 128 (2016) 512-520.

[28] V. Bhatia, B. Mulgrew, Non-parametric likelihood based channel estimator for Gaussian mixture noise, Signal Process. 87 (11) (2007) 2569-2586.

[29] R.J. Kozick, B..M. Sadler, Maximum-likelihood array processing in non-Gaussian noise with Gaussian mixtures, IEEE Trans. on Signal Process. 48 (12) (2000) 3520-3535.

[30] A.N. Bishop, B. Fidan, B.D.O. Anderson, et al., Optimality analysis of sensor-target geometries in passive localization: Part 1-Bearing-only localization, in: 3rd International Conference on Intelligent Sensors, Sensor Networks and Information, (ISSNIP), IEEE, 2007, pp. 7-12.

[31] M. Gavish, A.J. Weiss, Performance analysis of bearing-only target location algorithms, IEEE Trans. Aero. Electron. Syst. 28 (3) (1992) 817-828.

[32] Y. Zhang, N. Meratnia, P. Havinga, Outlier detection techniques for wireless sensor networks: A survey, IEEE Comm. Surv. Tutorials 12 (2) (2010) 159-170.

[33] P.J. Huber, Robust regression: asymptotics, conjectures and Monte Carlo, Ann. Stat. (1973) 799-821.

[34] D.E. Tyler, Robust statistics: theory and methods, (2008) 888-889.

[35] P. Guillaume, R. Pintelon, A Gauss-Newton-like optimization algorithm for" weighted" nonlinear least-squares problems, IEEE Trans. Signal Process. 4 (9) (1996) 2222-2228.

[36] M. Grant, S. Boyd, Y. Ye, CVX: Matlab Software for Disciplined Convex Programming, 2012. ver. 2.0 Beta, Sep[Online]. Available http://cvxr.com/cvx.

[37] S Boyd, L. Vandenberghe, Convex Optimization, Cambridge University Press, 2004. 